\title{
BMJ Open Identifying the top research priorities in postmastectomy breast cancer reconstruction: a James Lind Alliance priority setting partnership
}

\author{
Toni Zhong (D) , ${ }^{1}$ Anisha Mahajan, ${ }^{1}$ Katherine Cowan, ${ }^{2,3}$ Claire Temple-Oberle, ${ }^{4}$ \\ Geoff Porter, ${ }^{5}$ Martin LeBlanc, ${ }^{6}$ Kelly Metcalfe ${ }^{7}$
}

To cite: Zhong T, Mahajan A, Cowan $\mathrm{K}$, et al. Identifying the top research priorities in postmastectomy breast cancer reconstruction: a James Lind Alliance priority setting partnership. BMJ Open 2021;11:e047589. doi:10.1136/ bmjopen-2020-047589

- Prepublication history and additional supplemental material for this paper are available online. To view these files, please visit the journal online. (http://dx.doi.org/10.1136/ bmjopen-2020-047589).

Received 07 December 2020 Accepted 29 July 2021

Check for updates

(C) Author(s) (or their employer(s)) 2021. Re-use permitted under CC BY-NC. No commercial re-use. See rights and permissions. Published by BMJ.

For numbered affiliations see end of article.

Correspondence to

Dr Toni Zhong;

toni.zhong@uhn.ca

\section{ABSTRACT}

Background Major improvements in breast cancer treatment in the last decade include advancements in postmastectomy breast reconstruction (PMBR). Unfortunately, the studies in PMBR are primarily researcher or industry led with minimal input from patients and caregivers. The aim of this study is to use the James Lind Alliance (JLA) approach to bring together the patients, caregivers and clinicians in a priority setting partnership to identify the most important unanswered research questions in PMBR.

Methods The JLA priority setting methodology involved four key stages: gathering research questions on PMBR from patients, caregivers and clinicians; checking these research questions against existing evidence; interim prioritisation and a final consensus meeting to determine the top 10 unanswered research questions using the modified nominal group methodology.

Results In stage 1, 3168 research questions were submitted from 713 respondents across Canada, of which $73 \%$ of the participants were patients or caregivers. Stage 2 confirmed that there were a total of 48 unique unanswered questions. In stage three, 488 individuals completed the interim prioritisation survey and the top 25 questions were taken to a final consensus meeting. In the final stage, the top 10 unanswered research questions were determined. They cover a breadth of topics including personalised surgical treatment, safety of implants and newer techniques, access to PMBR, breast cancer recurrence and rehabilitation.

Interpretation Identification of the top 10 unanswered research questions is an important first step to generating relevant and impactful research that will ultimately improve the PMBR experience for patients with breast cancer.

\section{INTRODUCTION}

Breast cancer is the most common form of cancer among women in Canada ${ }^{1}$ and worldwide. $^{2}$ It was estimated that approximately 26900 Canadian women were diagnosed with breast cancer in 2019 which accounts for $25 \%$ of all cancer cases. ${ }^{1}$ With improvements in the detection and treatment of breast cancer, the

\begin{aligned} STRENGTHS AND LIMITATIONS OF THIS STUDY \\ \hline$\Rightarrow$ Use of the James Lind Alliance (JLA) approach to \\ bring together the patients, caregivers and clinicians \\ in a priority setting partnership to identify the most \\ important unanswered research questions in post- \\ mastectomy breast reconstruction (PMBR). \\ $\Rightarrow$ The JLA priority setting methodology involved four \\ key stages: gathering research questions on PMBR \\ from patients, caregivers and clinicians; check- \\ ing these research questions against existing evi- \\ dence; interim prioritisation and a final consensus \\ meeting to determine the top 10 unanswered re- \\ search questions using the modified nominal group \\ methodology. \\ $\Rightarrow$ Eighty-nine per cent of the participants who submit- \\ ted an uncertainty were under 65 years old; it shows \\ that older patients' may have not been included. \\ $\Rightarrow$ Eight-eight per cent of the survey participants were \\ of European, Canadian or American descent, greatly \\ under-representing the ethnic diversity in Canada. \\ $\Rightarrow$ While the top 10 uncertainties were important to \\ direct future research efforts, the more comprehen- \\ sive list of all unanswered research questions are \\ equally informative. \end{aligned}

5-year survival rate has increased to $88 \%$ for this disease. ${ }^{3}$ It is, therefore, imperative that breast cancer survivorship research broadens its scope to include areas such as postmastectomy breast reconstruction (PMBR) that may combat adverse effects following cancer surgery in the breast cancer survivorship period. Increased patient demand for PMBR, ${ }^{4}$ concerns with the low level of evidence for many PMBR techniques and the inequitable access to PMBR across Canada ${ }^{5}$ have highlighted the urgent need for better research to inform patients, providers and policy-makers about optimal practices in PMBR.

Engaging patients in the research process allows patients to add their perspective as 'experts' from their unique experience through living with an illness, as well as their 
personal knowledge with treatments and the healthcare environment. ${ }^{6-9}$ To improve patient outcomes and to reduce the 'costly mismatch of research-to-needs', ${ }^{10}$ we (The National Integrated Consortium for Breast Reconstruction Research is composed of general and reconstructive surgeons from each province, a patient advisory panel, breast cancer organisations (Canadian Partnership Against Cancer, Canadian Breast Cancer Foundation) and other key stakeholders (Canadian Association of Provincial Cancer Agencies)) initiated the National Breast Cancer Reconstruction Priority Setting Partnership and followed the James Lind Alliance (JLA) method. JLA uses an established and rigorous approach to identify the treatment and management priorities of stakeholders (patients, caregivers, clinicians and support organisations) so they can be incorporated into research agendas. ${ }^{12}$ The goal of the National Breast Cancer Reconstruction Priority Setting Partnership was to identify the most important unanswered research questions in breast cancer reconstruction from the joint perspectives of patients, caregivers, clinicians and support organisations across Canada. ${ }^{13} 14$

\section{METHODS}

\section{Establishing a steering group}

In 2016, we ${ }^{1}$ formed the National Breast Cancer Reconstruction Priority Setting Partnership to identify the top research priorities in breast reconstruction. We formed a steering group composed of five breast reconstruction patients, five plastic surgeons, one general surgeon, one nurse, two community partners who were also breast cancer survivors, one JLA advisor and one project coordinator. The steering group was tasked with overseeing the partnership by taking part in bimonthly meetings and making critical decisions at key intervals of the priority setting partnership.

\section{The consultative process}

We received ethics approval from the University Health Network's research ethics board in January 2017. The first phase of the priority setting approach was to gather questions from participants using a survey. We created a website to host our survey and sent out the website link for our partner organisations to distribute within their networks. We invited prospective participants through advertisements sent through email blasts, social media posts, postcards and Google advertisements.

\section{Gathering uncertainties}

We circulated an online survey, delivered in English and French, between 1 May and 31 August 2018 (online supplemental appendix 1). We promoted the survey through partner organisations' websites, and their social media platforms. We mailed postcards out to surgeons in five Canadian provinces to be distributed to their patients. We sent out email blasts to members of clinical associations, such as the Canadian Society of Plastic Surgeons,
Canadian Nurses Association and the Aboriginal Nurses Association of Canada. We also sent out email blasts to breast cancer survivors who attended the Breast Cancer Awareness events held throughout Canada. We placed an advertisement in four Canadian newsletters and we created google advertisements to target a demographic that keyed in phrases such as 'breast reconstruction' or 'breast cancer'. Through these advertisements, we targeted breast cancer survivors, their caregivers and the clinicians who treat them to participate however; there was no way of authenticating the survey participants' identity.

\section{Patient and public involvement}

The JLA priority setting methodology ensures patient and other key stakeholder involvement in each step of the research process from active participation in the research steering group, to gathering the initial research questions or uncertainties in PBMR, design of the initial and interim survey instruments, data analysis and verification of uncertainties as well as participation in the final priority setting consensus workshop. Results will be disseminated to participants through the JLA and patient experts have been thanked in the acknowledgements section of this report.

\section{Data analysis and verifying uncertainties}

We downloaded the English and French (these generated 3168 uncertainties in total) online survey results through SurveyMonkey and exported them into Microsoft Excel. The French responses were translated into English using professional paid interpreter from University Health Network's translation services. The French responses (271 uncertainties) were included into the overall survey and if there was any discrepancy in the translation, this was reviewed with the interpreter over email. Overall, there were no concerns with amalgamation of the translated responses from French through translation services.

We removed patient and clinician identifiers to maintain anonymity. Our health information specialist (AM) and PSP lead (TZ) grouped the questions into themes. Each theme was analysed by two members of the steering group, one with patient experience and the other being a healthcare professional. Questions were removed when both reviewers identified them as duplicates, unanswerable by research, out of scope or personal narratives rather than research questions. Discrepancies were settled by independent review by the PSP lead. Our health information specialist checked each in scope questions with existing guidelines, systematic reviews and clinical trials to ensure that our research questions have not been already fully answered with high-level evidence. For the literature review, there was a total of 36 articles that were shortlisted from our search strategy and 17 articles were deleted. The primary reason for deletion of the articles was irrelevance to the research study. The remaining articles were matched with the 48 researchable questions. Each question was colour coded to indicate whether it was 
'answered'; 'partially answered' or 'unanswered' through the research literature. Most of the questions fell within the latter two categories. The articles were summarised and a decision on whether to include the priority question was based on overall feedback from the available evidence, the consultative process and expert guidance of the study chair and steering committee members in entirely.

\section{Interim priority setting}

Questions that we labelled 'unanswered' were carried forward to the interim priority setting stage. Between 6 May 2019 and 14 June 2019, the second online survey asked participants to select and rank the top 10 uncertainties from the list of 48 uncertainties that we presented to them (online supplemental appendix 2). We presented the questions in random order to each survey participant to reduce bias.

\section{Final priority setting}

The 25 top ranked questions were taken forward to the final face-to-face priority setting consensus meeting that took place on 2 July 2019 in Toronto, Canada. We invited volunteers from partner organisations, and individuals who took part in the online survey to participate in the meeting. From this group, our steering group selected 24 participants who were from varying socioeconomic and cultural backgrounds arising from geographically diverse areas throughout Canada. These participants included breast reconstruction patients, mastectomy patients who did not undergo PMBR, family members, caregivers, plastic and general surgeons, radiation and medical oncologists, nurses, a social worker and a physiotherapist. The workshop participants were divided into three groups, each with a balanced distribution of patients, caregivers and clinicians. Each group was asked to rank the treatment uncertainty questions through group discussions using the modified nominal group technique facilitated by an independent JLA advisor. All group rankings of the questions were aggregated and were brought to the whole group for discussion. By the end of the priority setting meeting, we reached consensus on the top 10 Canadian research priorities in PMBR.

\section{RESULTS}

In the question-gathering survey, figure 1, we received 3168 uncertainties from 713 respondents across Canada between May and August 2018. We received questions from all the provinces and territories across Canada with the exception of Northwest Territories, and the vast majority of participants were from Ontario, Alberta and British Columbia (57.9\%) as shown on table 1. Patients and caregivers made up $73 \%$ of all survey participants. Of all the participants who submitted a question on PMBR, $44.6 \%$ had previously undergone PMBR and $16.6 \%$ had previously undergone mastectomy only. Clinicians made up $21.1 \%$ and nurses accounting for $40 \%$ of all the survey participants.

Two thousand nine hundred and thirty-one submissions were in scope and 237 submissions were out-of-scope. The out-of-scope submissions included questions about breast reduction surgeries, lumpectomies, medical insurance coverage and narratives about the participant's experience or statistics on cancer and cancer surgery. Our health information specialist (AM) and PSP lead (TZ) grouped similar or duplicate questions together generating 86 summary questions. Questions were grouped together when they were focused on the same topic. For example, questions that centred around the issue of physiotherapy or rehabilitation or exercise were group together. While questions that asked about longevity of implants, safety of different implant fill material, and breast implant illness were groups together under the implant safety. The majority $(68 \%)$ of the questions were on treatment options and surgical techniques, what to expect before and after PMBR, and resources or information provided to patients prior to PMBR. Figure 2 depicts the frequency of in-scope uncertainties by category.

A total of 86 summary questions were checked against 36 retrieved systematic reviews, clinical trials and guidelines in the existing literature. We found that none of the 86 summary questions had been sufficiently answered by a systematic review, clinical trial or guideline in the existing literature. Collectively, the steering group found 40 of the 86 questions to be either not researchable or repeat questions, therefore, these questions were removed. At a separate meeting, the steering group reviewed the remaining 46 questions. Where appropriate we combined questions to form one uncertainty or separated broader questions into two or more uncertainties such that each uncertainty contained only one distinct researchable question. We had a total of 48 uncertainties that we included in the interim survey for prioritisation.

The 48 uncertainties were prioritised through an online survey and completed by 488 participants from across Canada. Patients and caregivers made up $86 \%$ of the participants. Twenty-five of the uncertainties from six of the nine categories were shortlisted and brought to the final face-to-face priority setting consensus workshop.

There were 22 stakeholders present at the final priority setting consensus workshop. This diverse group included 13 breast cancer survivors or family members or caregiver, and 9 healthcare professionals including plastic surgeons, surgical oncologists, a radiation oncologist, a medical oncologist, a nurse, a social worker and a physiotherapist. The 1-day workshop followed the JLA protocol and used nominal group technique to reach consensus. Participants were divided into two groups with an equal distribution of patients, caregivers and clinicians in each group. Each group was provided with a set of cards each with a different 'unanswered' research question, and each group ranked the 25 uncertainties. The rankings were the two groups were combined and all the workshop participants then came together to discuss these rankings. Areas 


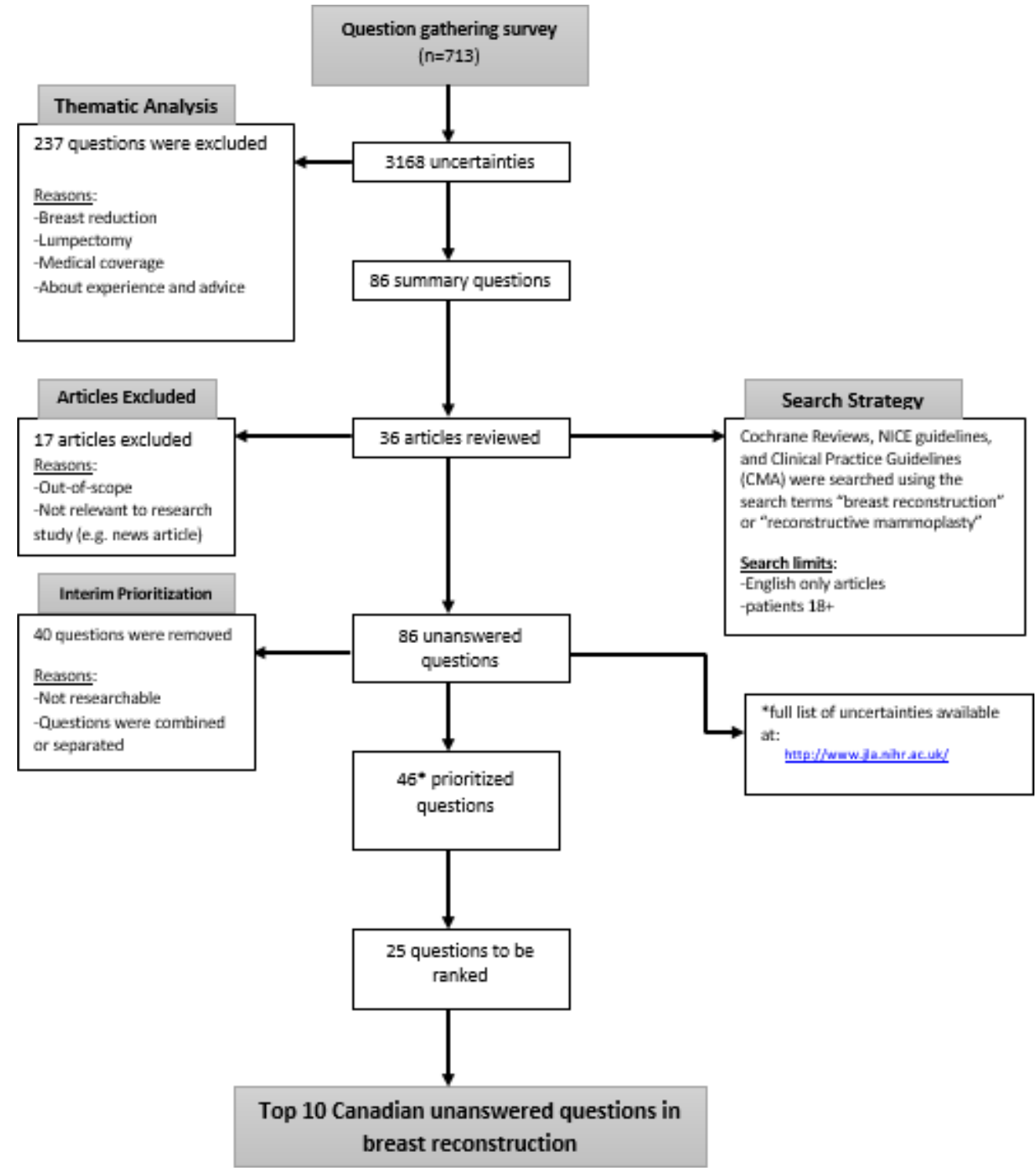

Figure 1 Flow diagram detailed (question gathering survey).

of disparity between the two groups were discussed openly and moderated by our JLA facilitator (KC). Following the open discussion, participants were reallocated into two new groups to consider the aggregate list of the 25 ranked questions. During the second round, there was a specific focus placed on the top 15 uncertainties, and the top 10 uncertainties were agreed by consensus by all the participants as listed on figure 3 .

\section{INTERPRETATION}

In this study, we used the JLA priority setting partnership process to identify the top 10 'treatment uncertainties' in the area of PMBR for which there are no up-to-date, reliable, systematic reviews of research evidence. This study highlighted the paucity of evidence-driven practice in PMBR care since none of the 86 research questions gathered from our survey have been previously answered by level I evidence. To address this problem, we formed a meaningful partnership among patients, caregivers, clinicians and support organisations to develop a consensus list of the top 10 unanswered research questions in PMBR. A limitation to our study methodology is that while the identification of the top 10 'treatment uncertainties' in the area of PMBR was achieved by incorporating the views of patients, caregivers and health professionals, 10 is an arbitrarily selected cut-off number and other important treatment questions were left off the list.

The uncertainties spanned a breadth of topics including personalised surgical treatment, safety of implants and newer techniques, access to care, cancer recurrence and rehabilitation. Identification of these questions is an important first step to generating relevant and impactful research that will ultimately improve the PMBR experience for patients with breast cancer. There were five major themes that emerged from the top 10 unanswered research questions. 
Table 1 Participant demographics

\begin{tabular}{|c|c|}
\hline Participant demographics & N, \% \\
\hline \multicolumn{2}{|l|}{ Patients and caregivers $\mathrm{N}=521$} \\
\hline Breast reconstruction patient & $318(61)$ \\
\hline Mastectomy patient & $119(23)$ \\
\hline Breast cancer patient & $53(10)$ \\
\hline Caregiver & $1(<1)$ \\
\hline $\begin{array}{l}\text { Patient/relative/friend of a breast recon, } \\
\text { mastectomy or breast cancer patient }\end{array}$ & $30(6)$ \\
\hline \multicolumn{2}{|l|}{ Clinicians N=145 } \\
\hline Nurse & $58(40)$ \\
\hline Physician & $10(7)$ \\
\hline Medical oncologist & $3(2)$ \\
\hline Radiation oncologist & $4(3)$ \\
\hline General surgeon & $16(11)$ \\
\hline Plastic surgeon & $24(16)$ \\
\hline Surgeon & $4(3)$ \\
\hline Physiotherapist & $24(16)$ \\
\hline Social worker & $2(1)$ \\
\hline \multicolumn{2}{|l|}{ Other $\mathrm{N}=14$} \\
\hline Mammography technologist & $1(7)$ \\
\hline BRCA positive gene carrier & $1(7)$ \\
\hline Advocate for awareness & $1(7)$ \\
\hline Psychologist & $2(14)$ \\
\hline Medical Imaging Technologist & $1(7)$ \\
\hline Student & $2(14)$ \\
\hline Healthcare professional & $1(7)$ \\
\hline Research & $4(28)$ \\
\hline Tattoo artist who does reconstruction tattoos & $1(7)$ \\
\hline \multicolumn{2}{|l|}{ Location N=692 } \\
\hline Alberta & $89(13)$ \\
\hline British Columbia & $78(11)$ \\
\hline Manitoba & $36(5)$ \\
\hline New Brunswick & $34(5)$ \\
\hline Newfoundland and Labrador & $26(4)$ \\
\hline Northwest Territories & $0(0)$ \\
\hline Nova Scotia & $38(5)$ \\
\hline Nunavut & $0(0)$ \\
\hline Ontario & $246(35)$ \\
\hline Prince Edward Island & $8(1)$ \\
\hline Quebec & $77(11)$ \\
\hline Saskatchewan & $59(8)$ \\
\hline Yukon & $1(<1)$ \\
\hline \multicolumn{2}{|l|}{ Ethnicity $n=692$} \\
\hline North American Aboriginal & $8(1)$ \\
\hline $\begin{array}{l}\text { Other North American (eg, Canadian or } \\
\text { American) }\end{array}$ & $305(44)$ \\
\hline
\end{tabular}

Continued
Table 1 Continued

\begin{tabular}{lc}
\hline Participant demographics & N, \% \\
\hline European & $304(44)$ \\
\hline Caribbean & $12(2)$ \\
\hline Latin, Central or South American & $4(<1)$ \\
\hline African & $9(1)$ \\
\hline Asian (eg, Middle Eastern, South, East and & $48(7)$ \\
Southeast Asian) & \\
\hline Oceania (eg, Australian) & $2(<1)$
\end{tabular}

Gender N=696

Female

Male $36(5)$

Other

Prefer not to say

$2(<1)$

Age $\mathrm{N}=699$

$\begin{array}{lc}15-24 & 5(<1) \\ 25-34 & 38(5) \\ 35-44 & 139(20) \\ 45-54 & 236(34) \\ 55-64 & 192(27) \\ 65+ & 81(11) \\ \text { Prefer not to say } & 8(1)\end{array}$

${ }^{*} \mathrm{~N}=$ number; values presented in the table are the number of respondents; proportions are included in the brackets. Not all respondents answered all questions.

BRCA, BReast CAncer gene.

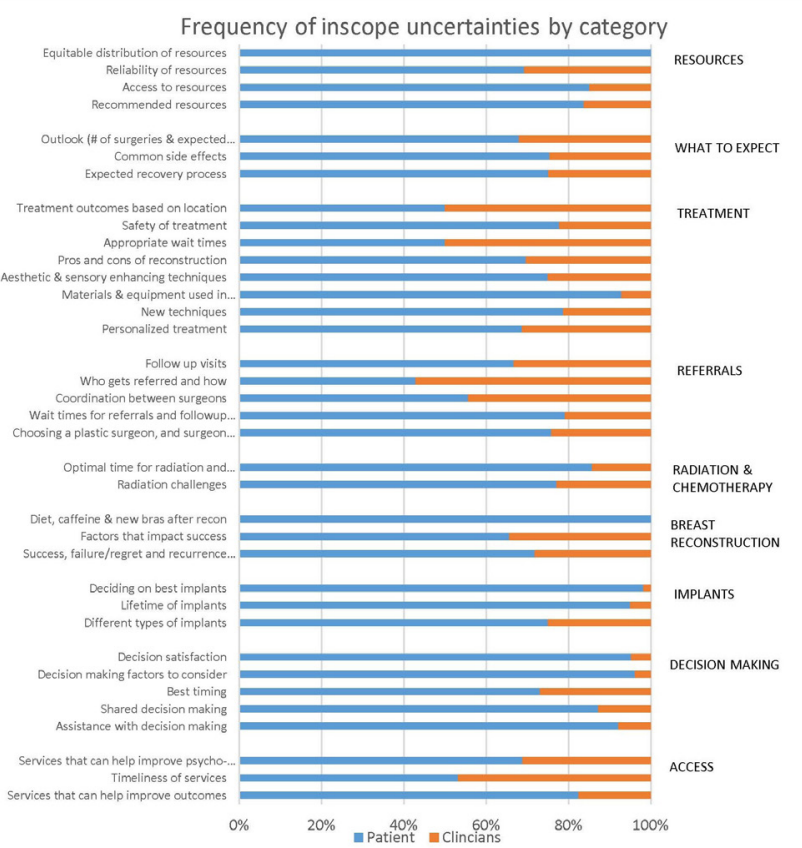

Figure 2 Frequency of in scope uncertainties by category. 


\section{Top 10 Research Priorities in Breast Reconstruction}
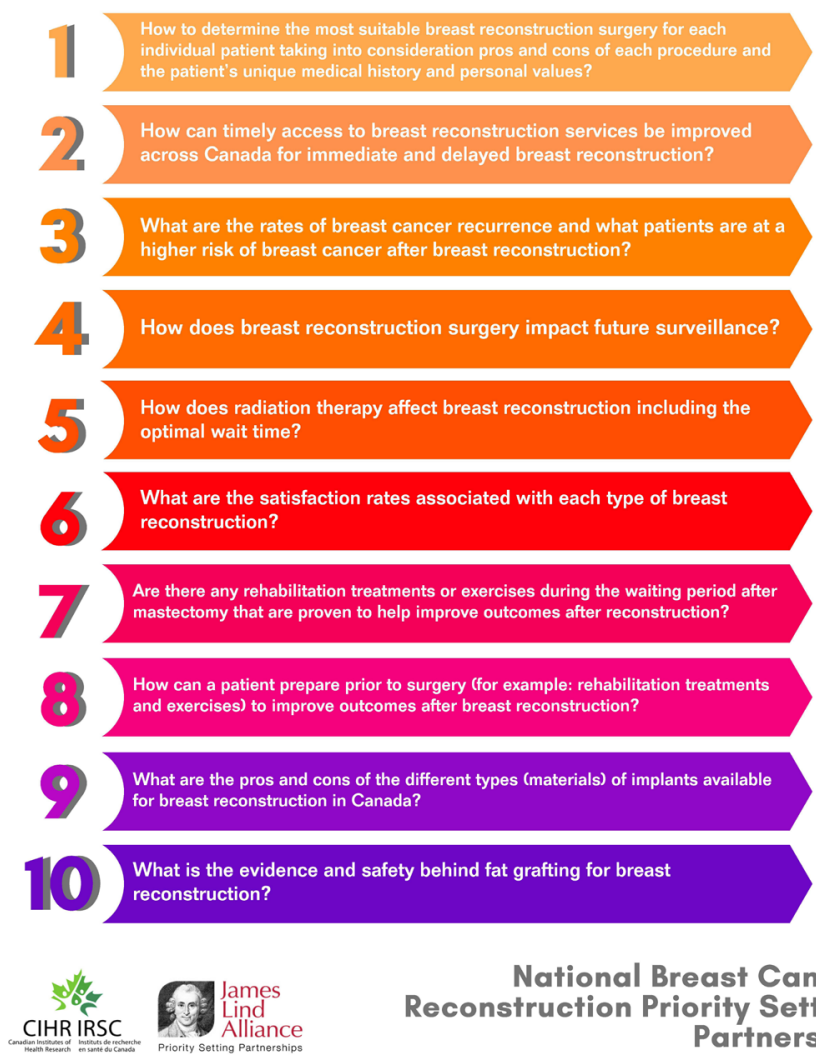

National Breast Cancer Reconstruction Priority Setting Partnership

Figure 3 Top 10 research priorities.

\section{Theme 1: personalised surgical care}

Patients, caregivers, clinicians and support organisations are most interested in research that can help determine the most suitable breast reconstruction procedure taking into account both the pros and cons of each surgical procedure and the unique characteristics and demands of each individual patient (Treatment Uncertainty Priority \#1). In keeping with the idea of personalised surgical care, our study showed that determining the 'right' match between the specific PMBR procedure and the individual patient was the most important consensus research question reached by the group. Our study also highlighted the need for research that examines patient satisfaction as an important measure of success (Treatment Uncertainty Priority \#6). It is interesting that other traditional endpoints or measures of surgical success such as complication rates or reoperation rates were not found to be as important in the priority setting partnership process.

\section{Theme 2: safety of implants and newer techniques}

We found that treatment uncertainties around the safety of breast implants was important to our study participants, in particular the pros and cons of different implant materials such as saline vs silicone gel (Treatment Uncertainty Priority \#9). Safety concerns with the use of newer techniques such as fat grafting or lipofilling to reconstructed breast (Treatment Uncertainty Priority \#10) was also highlighted to be an important treatment uncertainty. Our participants have also prioritised research that can better inform patients what the most optimal wait-time should be between radiotherapy to the chest and breast reconstruction to maximise the safety of PMBR. Currently, although there is a large body of research on the effects of radiation on PMBR, ${ }^{15} 16$ there are no published guidelines recommending the most optimal timing of delayed breast reconstruction after the completion of postmastectomy radiotherapy (Treatment Uncertainty Priority \#5).

\section{Theme 3: access}

The third theme was the need to address issues regarding the timely access to PMBR including both immediate and delayed reconstruction (Treatment Uncertainty Priority \#2). Our groups' research has shown that PMBR delivery in Canada is currently inequitable and in some areas inaccessible. The provincial rates of mastectomy with immediate PMBR (at same time as mastectomy) ranged from 7.6\% in Ontario between 2004 and 2010 to less than 2\% in Nova Scotia for breast cancer patients. ${ }^{17} 18$ These Canadian rates of PMBR are lower than the 29\% utilisation of PMBR reported by the Surveillance, Epidemiology and End Results database for the same time period in the United States. $^{19-21}$

\section{Theme 4: oncological safety}

The fourth theme that was identified to be a research priority for our group of patients, caregivers, clinicians and support organisations was the oncological safety of breast reconstruction on breast cancer recurrence and the impact of breast reconstruction on future surveillance of breast cancer (Treatment Uncertainty Priorities \#3 and \#4).

\section{Theme 5: rehabilitation}

The final theme of the treatment uncertainties prioritised by our group focused on the efficacy of rehabilitation to improve outcomes following breast reconstruction surgery. ${ }^{22-25}$ Both treatment uncertainty $\# 7$ and \#8 addressed research around interventions such as rehabilitation treatments or exercises that patients can undertake either following mastectomy or prior to breast reconstruction to optimise PMBR outcomes.

\section{CONCLUSION}

Since PMBR is increasingly recognised as an essential breast cancer survivorship issue for the 200000 mastectomy patients annually in North America, ${ }^{9}$ incorporating patient-oriented research in PMBR research can allow researchers to better address cancer patients' complex care needs. We have identified top 10 research priorities in PMBR using the rigorous JLA priority setting method. The most important aspect of this priority setting method was strengthening the relationship between patients, caregivers, clinicians and support organisations and generating a list of researchable questions valued by 
these stakeholders. Generating both reliable and up-todate research will help patients make a more informed and sound decision about PMBR, therefore, we hope that these results will guide future PMBR research to improve the relevancy of publications and research uptake.

\section{Author affiliations}

${ }^{1}$ Plastic Surgery, University Health Network, Toronto, Ontario, Canada

${ }^{2}$ Katherine Cowan Consulting Limited, St. Leonards-on-Sea, UK

${ }^{3}$ James Lind Alliance, Southampton, UK

${ }^{4}$ Plastic Surgery, Alberta Health Services Department of Surgery, Calgary, Alberta, Canada

${ }^{5}$ Canadian Partnership Against Cancer, Toronto, Ontario, Canada

${ }^{6}$ General Surgery, Dalhousie University Department of Surgery, Halifax, Nova Scotia, Canada

${ }^{7}$ University of Toronto, Toronto, Ontario, Canada

\section{Twitter Toni Zhong @drtonizhong}

Acknowledgements We would like to acknowledge all of the members of the Steering Committee including Clinicians: Dr. Amanda Roberts, Agnes Wisniewski and Dr. Perry Gdalevitch; Breast Cancer Survivors/ Community Partners: Natalie Witkin and Cathy Ammendolea; Patients or Caregivers: Dawn Eagleson, Jamie Mezzapelli, David Pawlik, Catherine Painvin and Maggie Easton.

Contributors $\mathrm{TZ}$ is responsible for all aspects of the study including analysis and interpretation of results, manuscript preparation and review. AM is responsible for data collection and analysis, interpretation of results and manuscript preparation. $\mathrm{KC}$ is responsible for data collection and analysis, interpretation of results and manuscript preparation. CT-0, ML, GP and KM are responsible for analysis and interpretation of results and manuscript preparation and review.

Competing interests 'Yes, there are competing interests for one or more authors and I have provided a Competing Interests statement in my manuscript and in the box below'

Patient consent for publication Not required.

Provenance and peer review Not commissioned; externally peer reviewed.

Data availability statement Data are available on reasonable request. All data relevant to the study are included in the article or uploaded as online supplemental information. Additional data are available upon request.

Supplemental material This content has been supplied by the author(s). It has not been vetted by BMJ Publishing Group Limited (BMJ) and may not have been peer-reviewed. Any opinions or recommendations discussed are solely those of the author(s) and are not endorsed by BMJ. BMJ disclaims all liability and responsibility arising from any reliance placed on the content. Where the content includes any translated material, BMJ does not warrant the accuracy and reliability of the translations (including but not limited to local regulations, clinical guidelines, terminology, drug names and drug dosages), and is not responsible for any error and/or omissions arising from translation and adaptation or otherwise.

Open access This is an open access article distributed in accordance with the Creative Commons Attribution Non Commercial (CC BY-NC 4.0) license, which permits others to distribute, remix, adapt, build upon this work non-commercially, and license their derivative works on different terms, provided the original work is properly cited, appropriate credit is given, any changes made indicated, and the use is non-commercial. See: http://creativecommons.org/licenses/by-nc/4.0/.

ORCID iD

Toni Zhong http://orcid.org/0000-0002-6454-8150

\section{REFERENCES}

1 Statistics CCSAC on C. Breast Cancer Statistics [Internet]. Canadian Cancer Statistics, 2019. Available: https://www.cancer.ca/en/cancer- information/cancer-type/breast/statistics/?region=on [Accessed 15 Oct 2019].

2 Panchal H, Matros E. Current trends in postmastectomy breast reconstruction. Plast Reconstr Surg 2017;140:7S-13.

3 Statistics CCSAC on C. Survival Statistics for Breast Cancer [Internet]. Canadian Cancer Statistics, 2019. Available: https://www. cancer.ca/en/cancer-information/cancer-type/breast/prognosis-andsurvival/survival-statistics/?region=on

4 Koshi EB, Andreou P, Morris SF. Breast reconstruction in nova Scotia: rate, trends and influencing factors. Can J Plast Surg 2003;11:135-40.

5 Platt J, Baxter N, Zhong T. Breast reconstruction after mastectomy for breast cancer. CMAJ 2011;183:2109-16.

6 Canadian Institutes of Health Research(ClHR). Strategy for PatientOriented Research - ClHR. Canadian Institutes of Health Research, 2016.

7 Bishop AC, Elliott MJ, Cassidy C. Moving patient-oriented research forward: thoughts from the next generation of knowledge translation researchers. Res Involv Engagem 2018;4:23.

8 Nixon NA, Simmons C, Lemieux J, et al. Research priorities in metastatic breast cancer: a James Lind alliance priority setting partnership. Breast J 2020;26:488-93.

9 Whelan T, Levine M. More evidence that locoregional radiation therapy improves survival: what should we do? J Natl Cancer Inst 2005;97:82-4.

10 Oliver S, Armes DG, Gyte G. Public involvement in setting a national research agenda: a mixed methods evaluation. Patient 2009;2:179-90.

11 Tallon D, Chard J, Dieppe P. Relation between agendas of the research community and the research consumer. Lancet 2000;355:2037-40.

12 Alliance JL. The James Lind alliance Guidebook: version 8. United Kingdom: The James Lind Alliance, 2018: 81.

13 Chan Errol Wei'en, Li X, Tham Y-C, et al. Glaucoma in Asia: regional prevalence variations and future projections. Br J Ophthalmol 2016;100:78-85.

14 Crowe S. Setting Priorities for Treatment Uncertainties- A Review of Methods [Internet], 2009. Available: http://www.jla.nihr.ac.uk/newsand-publications/downloads/review-of-priority-setting-methodssally-crowe.pdf

15 Yun JH, Diaz R, Orman AG. Breast reconstruction and radiation therapy. Cancer Control 2018:25:107327481879548.

16 Lin A, Christensen J, Liao E. Abstract 70: the impact of postmastectomy radiation therapy on permanent implants in Direct-toImplant breast reconstruction versus tissue expanders in two-stage breast reconstruction. Plast Reconstr Surg Glob Open 2018;6:55-6.

17 Zhong T, Spithoff K, Kellett S. Breast cancer reconstrution surgery (immediate and delayed) across Ontario: patient indications and appropriate surgical options. Toronto, 2016.

18 Porter G, Wagar B, Bryant $\mathrm{H}$, et al. Rates of breast cancer surgery in Canada from 2007/08 to 2009/10: retrospective cohort study. CMAJ Open 2014;2:E102-8.

19 Zhong T, Fernandes KA, Saskin R, et al. Barriers to immediate breast reconstruction in the Canadian universal health care system. $J$ Clin Oncol 2014;32:2133-41.

20 Karunanayake M, Bortoluzzi P, Chollet A, et al. Factors influencing the rate of post-mastectomy breast reconstruction in a Canadian teaching hospital. Plast Surg 2017;25:242-8.

21 Statistics CCSAC on C. Breast Reconstruction [Internet], 2019. Available: https://www.cancer.ca/en/cancer-information/cancertype/breast/reconstruction-and-prostheses/breast-reconstructionsurgery/?region=on

22 Eltahir Y, Werners LLCH, Dreise MM, et al. Quality-Of-Life outcomes between mastectomy alone and breast reconstruction. Plast Reconstr Surg 2013;132:201e-9.

23 Thorarinsson A, Fröjd V, Kölby L, et al. Long-Term health-related quality of life after breast reconstruction: comparing 4 different methods of reconstruction. Plast Reconstr Surg Glob Open 2017:5:e1316.

24 Beugels J, Kool M, Hoekstra LT, et al. Quality of life of patients after immediate or delayed autologous breast reconstruction: a multicenter study. Ann Plast Surg 2018;81:523-7.

25 Webb C, Sharma V, Temple-Oberle C. Delivering breast reconstruction information to patients: women report on preferred information delivery styles and options. Plast Surg 2018;26:26-32. 